Cahiers
Recherche
sur les Droits

Fondamentaux

Cahiers de la recherche sur les droits

fondamentaux

4 | 2005

Quel avenir pour la laïcité cent ans après la loi de 1905 ?

\title{
La protection des religions minoritaires en droit international et européen
}

Jean-Manuel Larralde

\section{OpenEdition}

Journals

Édition électronique

URL : https://journals.openedition.org/crdf/7363

DOI : $10.4000 /$ crdf.7363

ISSN : 2264-1246

Éditeur

Presses universitaires de Caen

Édition imprimée

Date de publication : 1 décembre 2005

Pagination : 157-168

ISBN : 2-84133-250-0

ISSN : $1634-8842$

Référence électronique

Jean-Manuel Larralde, "La protection des religions minoritaires en droit international et européen », Cahiers de la recherche sur les droits fondamentaux [En ligne], 4 | 2005, mis en ligne le 15 décembre 2020, consulté le 14 novembre 2022. URL : http://journals.openedition.org/crdf/7363 ; DOI : https:// doi.org/10.4000/crdf.7363 


\title{
La protection des religions minoritaires en droit international et européen
}

\author{
Jean-Manuel LARRALDE \\ Maître de conférences à I'Université de Caen Basse-Normandie
}

I. La protection des religions minoritaires, composante implicite de la liberté de religion

A. Une protection induite par les normes internationales et européennes

B. Des jurisprudences prudentes

II. La protection des religions minoritaires comme composante de la notion de minorité nationale

A. Un ensemble normatif protecteur des minorités religieuses

B. Des techniques de protection de nature diplomatique

La religion et le respect des croyances ont longtemps constitué un sujet de controverses et de conflits, notamment en Europe, où l'idée de liberté religieuse n'est pas toujours allée de soi. Les groupes religieux minoritaires ont été persécutés dès l'Empire romain et le $\mathrm{xx}^{\mathrm{e}}$ siècle aura été celui du Génocide des juifs... Jusqu'au milieu du Xvi ${ }^{\mathrm{e}}$ siècle, l'idée de tolérance ne trouve en effet refuge que dans certains groupes spiritualistes minoritaires ou chez quelques rares penseurs, la célèbre Lettre sur la tolérance de John Locke, publiée en 1689, étant à ce titre souvent citée comme modèle du genre ${ }^{1}$. Certes, l'idée de liberté religieuse n'a pas toujours été absente dans l'histoire. La liberté de conscience était ainsi reconnue aux juifs et infidèles dans le monde médiéval. Elle comprenait même la liberté de culte, dans la mesure où elle n'était pas illégalement entravée par le fanatisme populaire ou par l'arbitraire des rois et des seigneurs. Par contre, l'intolérance médiévale à l'encontre des hérétiques et des schismatiques était absolue. Essentiellement fondée sur l'unité de la foi chrétienne (comme l'Islam lui-même était fondé en une foi absolue en Allah), la société médiévale se montrait totalement impitoyable à l'encontre de quelque forme d'hérésie que ce soit. Toute tentative de corrompre la foi ou de diviser l'unité chrétienne pouvait apparaître comme une véritable trahison. Mais les politiques étatiques se sont avérées longtemps rétives à la reconnaissance du pluralisme religieux. En France en particulier, est régulièrement mentionnée au début des temps modernes la maxime « une foi, une loi, un roi ${ }^{2}$. C'est en s'y référant que l'on aura énormément de mal à accepter les édits de tolérance. De même, le Toleration Act anglais de 1689 excluait les catholiques et les sociniens ${ }^{3}$. La paix d'Augsbourg (1555), transaction entre les princes luthériens et les princes catholiques, ne constitue pas davantage l'application d'un principe de tolérance religieuse : la formule Cujus regio, ejus religio... met en place une formule «territorialiste », qui n'admet qu'une confession par État et fait du prince le maître de la religion. Chaque prince peut librement choisir sa religion, luthérienne ou catholique, et l'imposer à ses sujets. Ce principe constitue la négation même de la liberté des

1. Locke exclut toutefois explicitement les "papistes» de la tolérance religieuse, car relevant d'un souverain étranger. Voir J. Lecler, M.-F. Valkhoff, Les Premiers Défenseurs de la liberté religieuse, Paris, Éditions du Cerf, 1969, vol. 1, p. 22.

2. Citée comme courante par le chancelier Michel de L'Hospital dans son discours d'ouverture aux États Généraux de 1560.

3. Doctrine hérétique de Lélius Socin, réformateur italien du Xvi siècle qui rejette La Trinité, la divinité de Jésus. 
cultes $^{4}$. Il faut attendre le grand mouvement d'indépendance des Pays-Bas à la fin du Xvi siècle pour qu'apparaisse l'idée d'assurer une liberté religieuse. La "paix de religion », dont le texte fut soumis en juillet 1578 par les États Généraux à l'approbation des provinces et des villes, rappelle que la consolidation des libertés acquises n'est possible que « si nous entrons en une plus étroite obligation, accord et union, mêmement au regard de la religion ».

Le principe d'une tolérance religieuse reconnaissant, sous limites, toutes religions et confessions au sein de l'État, apparaît donc comme une idée très récente, ce qui s'explique notamment par les caractéristiques propres des religions (et tout spécialement des religions monothéistes) : toute religion porte en elle une notion d'absolu qui aboutit à ce qu'elle n'admette aucun partage. L'Église catholique affirmait ainsi encore en 2000 qu' " on peut et on doit dire que Jésus-Christ a une fonction unique et singulière pour le genre humain et pour son histoire: cette fonction lui est propre, elle est exclusive, universelle et absolue ». Les conclusions en sont donc logiques: «La Vérité, qui est le Christ, s'impose comme une autorité universelle. [...] Le mystère chrétien dépasse en effet toute limite d'espace et de temps; il réalise l'unité de la famille humaine ${ }^{5}$. Ces visions hégémoniques de la religion et de la croyance laissent toutefois progressivement la place au $\mathrm{xx}^{\mathrm{e}}$ siècle à une nouvelle conception de la liberté religieuse, notion forgée par les différents textes internationaux et européens, et qui s'apparente désormais davantage à une liberté plurale de croyance, de pensée et de religion. La liberté de religion est donc une liberté concurrentielle, qui permet à des religions que l'on peut qualifier de «minoritaires » d'exister à côté de la religion de la majorité de la population (cette religion pouvant d'ailleurs avoir, ou non, le statut de religion d'État ou de religion officielle). Mais la notion même de "religion minoritaire » apparaît relativement délicate à cerner. En effet, ce même concept renvoie tout à la fois aux croyants relevant de religions ou de rites qui ne sont pas ceux de la majorité de la population de l'État. Mais il peut également s'agir de la religion pratiquée par des personnes appartenant à des groupes plus homogènes. La religion minoritaire sera alors l'un des éléments constitutifs d'une minorité nationale. Même si le droit n'a encore aujourd'hui jamais réellement consacré la notion de « religion minoritaire » dans l'une ou l'autre des deux acceptions, la perspective « ouverte» de la liberté de religion permet la reconnaissance d'une pluralité religieuse, comme composante inhérente à ce droit fondamental (I). En outre, par l'intermédiaire d'une protection internationale des minorités, le droit va se faire l'acteur d'une protection des religions minoritaires, c'est-à-dire des religions et des croyances comme composantes mêmes du fait minoritaire (II).

\section{La protection des religions minoritaires, composante implicite de la liberté de religion}

Le concept même de « religion minoritaire » n'a jamais été consacré par les normes supranationales, mais l'approche globalisante de la liberté de religion par ces mêmes textes aboutit à ce que l'on puisse parler d'une protection de ces groupes de croyants «induite» par les textes (A). Ce type de protection implicite se trouve renforcé par la jurisprudence supranationale, même si les zones d'ombres et interrogations liées à ce corps de jurisprudence n'ont pas toutes été levées (B).

\section{A. Une protection induite par les normes internationales et européennes}

La liberté de religion figure indéniablement parmi les éléments du «noyau dur » des droits internationalement protégés. Mais il s'agit d'une liberté au champ d'application particulièrement délicat à définir, car elle incorpore plusieurs éléments de protection qui se combinent ${ }^{6}$. L'exercice de la religion laisse tout d'abord apparaître deux champs d'application. Le premier renvoie à une liberté individuelle qui consiste pour toute personne «à donner ou non son adhésion personnelle à une religion, à la choisir librement ou à la refuser ». Il s'agit, en outre, d'une liberté collective « en ce sens que, ne s'épuisant pas dans la foi ou dans la croyance elle [la religion] donne nécessairement naissance à une "pratique" dont le libre exercice doit être garanti » ${ }^{7}$. La liberté de religion est, en outre, une liberté « synthèse » qui comprend, tout à la fois, une liberté de conscience, de croyance ou de conviction, mais également un droit à manifester et à extérioriser ses convictions (ou à ne pas le faire $\left.^{8}\right)$. Enfin, elle signifie, théoriquement, un droit à changer de conviction, qui ne peut en aucun cas être imposé à l'intéressé. La protection de la liberté religieuse par les normes supranationales offre donc l'image d'un ensemble complexe, tant en ce qui concerne sa définition que son champ d'application.

Dès le troisième projet Wilson de Pacte pour la Société des Nations du 20 janvier 1919 figure une mention relative au libre exercice de la religion et l'égalité, en droit et en fait,

4. L'histoire des principautés germaniques montrera à plusieurs reprises que la tolérance religieuse peut être supprimée par un prince et rétablie par un autre... On peut également conjuguer la tolérance religieuse avec des règles drastiques qui limitent en pratique les libertés des Églises (comme le démontre le Toleranzpatent de l'empereur Joseph II en 1781). Cf. J. Lecler, M.-F. Valkhoff, Les Premiers Défenseurs de la liberté religieuse, vol. 2, p. 160.

5. Congrégation pour la doctrine de la foi, Déclaration «Dominus Jesus» sur l'unicité et l'universalité salvifique de Jésus-Christ et de l’Église, 5 septembre 2000 .

6. L'article 6 de la Résolution 36 / 55, proclamée par l'Assemblée générale des Nations Unies le 25 novembre 1981, présente l'énumération la plus précise, en neuf points, des libertés induites par la liberté de religion.

7. J. Robert, La Liberté religieuse et le régime des cultes, Paris, PUF, 1977, p. 3.

8. Certaines juridictions constitutionnelles utilisant d'ailleurs le concept de «liberté religieuse sous son aspect négatif». Cour constitutionnelle fédérale allemande, 16 mai 1995. Conseil de l'Europe, Bulletin de jurisprudence constitutionnelle, édition spéciale « liberté confessionnelle», 2000, p. 12. 
des adhérents des différents cultes ${ }^{9}$. Cette disposition ne sera toutefois pas reprise dans le texte définitif et il faut attendre l'adoption de la Déclaration universelle des droits de l'homme du 10 décembre 1948 pour que soit proclamé le droit de toute personne « à la liberté de pensée, de conscience et de religion; ce droit implique la liberté de changer de religion ou de conviction ainsi que la liberté de manifester sa religion ou sa conviction seul ou en commun, tant en public qu'en privé, par l'enseignement, les pratiques, le culte et l'accomplissement des rites » (art. 18). Une formulation proche est ensuite reprise par les plus grands textes conventionnels, tels l'article 18 du Pacte sur les droits civils et politiques du 16 décembre $1966^{10}$ et l'article 5-dVII de la Convention internationale sur l'élimination de toutes les formes de discrimination raciale du 21 décembre $1965^{11}$. La liberté de religion constitue également une préoccupation pour plusieurs textes spécifiques, tels que la Convention relative au statut des réfugiés du 28 juillet $1951^{12}$, l'article 14 de la Convention relative aux droits de l'enfant du 26 janvier $1990^{13}$, ou encore la Convention internationale sur la protection des droits de tous les travailleurs migrants et des membres de leur famille du 18 décembre $1990^{14}$. La liberté de religion n'est pas davantage absente des textes régionaux, tels que l'article 2 de la Convention américaine relative aux droits de l'homme du 22 novembre $1969^{15}$, l'article 9 de la Convention européenne de sauvegarde des droits de l'homme et des libertés fondamentales du 10 novembre $1950^{16}$, ou encore, dans une formule plus concise, l'article 8 de la Charte africaine des droits de l'homme et des peuples du 27 juin $1981^{17}$.

La liberté de religion apparaît donc indéniablement comme l'un des éléments essentiels des droits fondamentaux internationalement protégés. Néanmoins, l'étude du seul instrument international protégeant de manière spécifique la liberté de religion peut apparaître fort décevante de prime abord. La Déclaration sur l'élimination de toutes les formes d'intolérance et de discrimination fondées sur la religion ou la conviction de 1981, issue d'un long processus d'élaboration initié en $1956^{18}$, apparait comme un texte de compromis, apportant fort peu par rapport aux normes préexistantes. Ce texte fait de la liberté de religion ou de conviction l'un des éléments fondamentaux de la vie de chacun. Cette liberté de religion doit, en outre, permettre de contribuer à la réalisation des buts de paix mondiale, de justice sociale et d'amitié entre les peuples. La Déclaration ne constitue pas un instrument de reconnaissance officielle des religions minoritaires, mais elle prohibe toutefois toute discrimination fondée sur la religion ou la conviction (art. 3 et 4). Il faut surtout souligner que ce texte ne constitue qu'une norme de soft law, tout

9. «Reconnaissant que les persécutions et l'intolérance religieuses sont des causes fécondes de guerre, les Puissances signataires conviennent - et la Société des Nations exigera de tous les nouveaux États, ainsi que de tous les États demandant leur admission dans son sein, une promesse au même effet - qu'elles n'édicteront aucune législation interdisant ou entravant le libre exercice de la religion, et qu'elles ne prendront aucune mesure d'un caractère différentiel, ni en droit ni en fait, au détriment de ceux qui pratiquent une foi, une religion ou une croyance particulières, dont les rites ne sont pas incompatibles avec l'ordre public, et les bonnes mœurs" (arrangement $n^{\circ} 6$ ).

10. «1. Toute personne a droit à la liberté de pensée, de conscience et de religion ; ce droit implique la liberté d'avoir ou d'adopter une religion ou une conviction de son choix, ainsi que sa liberté de manifester sa religion ou sa conviction, individuellement ou en commun, tant en public qu'en privé, par le culte et l'accomplissement des rites, les pratiques et l'enseignement. 2. Nul ne subira de contrainte pouvant porter atteinte à sa liberté d'avoir ou d'adopter une religion ou une conviction de son choix. 3. La liberté de manifester sa religion ou ses convictions ne peut faire l'objet que des seules restrictions prévues par la loi et qui sont nécessaires à la protection de la sécurité, de l'ordre et de la santé publique, ou de la morale ou des libertés et droits fondamentaux d'autrui. 4. Les États parties au présent Pacte s'engagent à respecter la liberté des parents et, le cas échéant, des tuteurs légaux de faire assurer l'éducation religieuse et morale de leurs enfants conformément à leurs propres convictions ».

11. «Les États parties s'engagent à interdire et à éliminer la discrimination raciale sous toutes ses formes et à garantir le droit de chacun à l'égalité devant la loi sans distinction de race, de couleur ou d'origine nationale ou ethnique, notamment dans la jouissance des droits suivants [...] : droit à la liberté de pensée, de conscience et de religion».

12. Son article 4 dispose que «les réfugiés bénéficieront d’un traitement au moins aussi favorable que celui accordé aux nationaux en ce qui concerne la liberté de pratiquer leur religion et en ce qui concerne la liberté d'instruction religieuse de leurs enfants », et l'article 33 interdit qu'un réfugié soit expulsé ou refoulé vers un pays où sa vie ou sa liberté seraient menacées en raison de sa religion.

13. «1. Les États parties respectent le droit de l'enfant à la liberté de pensée, de conscience et de religion. 2. Les États parties respectent le droit et le devoir des parents ou, le cas échéant, des représentants légaux de l'enfant, de guider celui-ci dans l'exercice du droit susmentionné d'une manière qui corresponde au développement de ses capacités. 3. La liberté de manifester sa religion ou ses convictions ne peut être soumise qu'aux seules restrictions qui sont prescrites par la loi et qui sont nécessaires pour préserver la sûreté publique, l’ordre public, la santé et la moralité publiques, ou les libertés et droits fondamentaux d'autrui ».

14. En particulier l'article 12 qui traite du droit des travailleurs migrants et des membres de leur famille à la liberté de pensée, de conscience et de religion.

15. «Toute personne a droit à la liberté de conscience et de religion. Ce droit implique la liberté de garder sa religion ou ses croyances ou de changer de religion ou de croyances ainsi que la liberté de professer et de répandre sa foi ou ses croyances individuellement ou collectivement en public ou en privé. Nul ne peut être l'objet de mesures de contraintes de nature à restreindre sa liberté de garder sa religion ou ses croyances ou de changer de religion ou de croyances. La liberté de manifester sa religion ou ses croyances ne peut faire l'objet d'autres restrictions que celles qui, prévues par la loi, sont nécessaires à la sécurité publique, à la protection de l'ordre, de la santé ou de la morale publics; à la sauvegarde des droits ou libertés d'autrui. Les parents et, le cas échéant, les tuteurs ont droit à ce que leurs enfants et pupilles reçoivent l'éducation religieuse conforme à leurs propres convictions ».

16. «1. Toute personne a droit à la liberté de pensée, de conscience et de religion; ce droit implique la liberté de changer de religion ou de conviction, ainsi que la liberté de manifester sa religion ou sa conviction individuellement, en public ou en privé, par le culte, l'enseignement, les pratiques et l'accomplissement des rites. 2. La liberté de manifester sa religion ou ses convictions ne peut faire l'objet d'autres restrictions que celles qui, prévues par la loi, constituent des mesures nécessaires, dans une société démocratique, à la sécurité publique, à la protection de l'ordre, de la santé ou de la morale publiques, ou à la protection des droits et libertés d'autrui».

17. «La liberté de conscience, la profession et la pratique libre de la religion sont garanties. Sous réserve de l'ordre public, nul ne peut être l'objet de mesures de contrainte visant à restreindre la manifestation de ces libertés ».

18. Résolution 36 / 55 proclamée par l'Assemblée générale des Nations Unies le 25 novembre 1981. Voir E. Odio Benito, « Historique de la liberté religieuse et de la Déclaration sur l'élimination de toutes les formes d'intolérance et de discrimination fondées sur la religion ou la conviction », Conscience et Liberté, $\mathrm{n}^{\circ} 30,1985$, p. 40 sq. 
instrument conventionnel en la matière apparaissant, encore aujourd'hui, largement improbable.

Aucun de ces textes ne mentionne toutefois les religions minoritaires, ni dans le cadre de la liberté de religion, ni dans le cadre de relations avec une religion majoritaire. Toutefois, la complexité de la liberté de religion ${ }^{19}$ offre, implicitement, la voie à la reconnaissance des religions minoritaires, car si la protection de telles religions ou croyances n'apparaît jamais au sein des droits fondamentaux internationalement protégés, on ne peut toutefois que reconnaître une reconnaissance " en creux » par les textes. La proclamation de la liberté religieuse, liée à la prohibition de toute discrimination, permet en effet de protéger les groupes religieux minoritaires et leurs adeptes. En outre, la volonté, plus récente, de lutte contre l'intolérance religieuse apparaît comme étant de nature à renforcer l'intensité de cette protection supranationale.

En premier lieu, les différentes normes supranationales, qui protègent la liberté de religion, protègent également dans certains cas la liberté de changer de religion ou de conviction. Cette possibilité est protégée de manière explicite par l'article 18 de la Déclaration universelle et l'article 9 de la Convention européenne des droits de l'homme. En indiquant la possibilité de changer de religion ou de conviction, ces textes condamnent les systèmes où l'État imposerait une religion officielle et interdirait toute idée de pluralisme dans le domaine religieux ${ }^{20}$. Cette possibilité de pouvoir changer librement de religion ou de conviction implique évidemment que les individus ne se voient imposer ni culte d'État, ni ne soient pénalisés par des dispositifs répressifs interdisant par exemple l'apostasie. Il convient cependant de remarquer que d'autres instruments restent plus discrets sur ces possibilités de changement de religion. Ainsi la Déclaration onusienne de 1981 ne prévoit pas ce droit, tout comme la Déclaration islamique universelle du 19 septembre $1981 . .$. On peut d'ailleurs relever plus généralement qu'aucun des textes régionaux non européens ne prévoit la possibilité explicite de changer de religion. Ceci résulte de l'opposition de certains États à religion officielle ou dominante et qui, bien loin de reconnaître la possibilité de changer de religion, la pénalisent plutôt en réprimant l'apostasie.

Bien qu'évidemment préoccupante, cette absence de mention explicite de la possibilité de changer de religion ne constitue pas un argument insurmontable quant à la protection des groupes religieux minoritaires. En effet, la protection de la liberté religieuse doit être associée, dans plusieurs instruments, à d'autres clauses qui prohibent la discrimination ${ }^{21}$. Cette possibilité existe notamment dans le cadre de la Convention européenne des droits de l'homme, avec son article 14, qui prohibe toute discrimination, fondée notamment sur la religion. La méconnaissance de l'article 14 peut aboutir à l'aggravation d'un autre droit protégé par la Convention. De même, l'article $26 \mathrm{du}$ Pacte sur les droits civils et politiques du 16 décembre 1966 (qui reprend le principe général d'égalité devant la loi tel que proclamé par l'article 7 de la Déclaration universelle des droits de l'homme de 1948) constitue une source de protection globale. Pour le Comité des droits de l'homme dans son observation générale $\mathrm{n}^{\circ} 18$ (37) relative à la nondiscrimination, l'article 26 prévoit en lui-même un droit autonome et interdit toute discrimination en droit ou en fait dans tout domaine réglementé et protégé par les pouvoirs publics. Par suite, lorsqu'un État adopte un texte législatif, il doit faire en sorte que son contenu ne soit pas discriminatoire. Le Comité des droits de l'homme est donc en mesure de constater l'incompatibilité du Pacte avec des législations ou pratiques nationales mettant en œuvre des dispositifs discriminatoires à l'encontre des religions minoritaires ${ }^{22}$. La mention du libre choix de la religion et de la possibilité de changer de religion ou de conviction s'oppose à l'idée de religion imposée par l'État ou de contrôle et d'interdiction générale des cultes et croyances ne relevant pas de la religion majoritaire. Cette optique large de protection de la liberté religieuse semble aujourd'hui également partagée par les normes régionales de protection des droits ${ }^{23}$.

Élément implicite de la liberté de religion, la protection des religions minoritaires se voit aujourd'hui renforcée par la volonté des organes onusiens d'inscrire cette

19. Que l'on trouve également développée dans certaines jurisprudences nationales: pour la Cour constitutionnelle autrichienne, le «droit de pratiquer les religions » comprend « la liberté dite de culte au sens étroit » (qui comprend les activités de croyance qui ont un rapport quel qu’il soit avec un événement tel qu'un service divin et qui supposent au moins la formation d'un culte primitif) et la « liberté de religion » (qui comprend toutes les autres formes des manifestations de croyance, que ce soient des comportements privés ou publics). Cour constitutionnelle autrichienne, 27 septembre 1950. Conseil de l'Europe, Bulletin de jurisprudence constitutionnelle, 2000, p. 31.

20. Voir D.J. Sullivan, «Advancing the freedom of religion or belief through the United Nations Declaration on the elimination of religious intolerance and discrimination ", American Journal of International Law, 1988, p. 487 sq.

21. Le principe de non-discrimination religieuse ayant également fait l'objet de plusieurs consécrations constitutionnelles en Europe, comme en Allemagne avec les articles 3-3 ( Nul ne doit être défavorisé ni privilégié en raison de [...] ses opinions religieuses ou politiques [...]») et 33-3 («La jouissance des droits civils et civiques, l'admission aux fonctions publiques, ainsi que les droits acquis dans la fonction publique sont indépendants de la croyance religieuse. Personne ne doit subir de préjudice en raison de son adhésion à une croyance religieuse et philosophique ») de la loi fondamentale du 23 mai 1949. Voir également (entre autres) l'article 16.3 de la Constitution espagnole du 27 décembre 1978, l'article 41 de la Constitution portugaise du 2 avril 1976, l'article 11 de la Constitution belge du 17 février $1994 \ldots$

22. Encore faudrait-il toutefois que les États les plus concernés aient ratifié le Pacte international relatif aux droits civils et politiques et surtout reconnu la compétence du Comité des droits de l'homme pour connaître des communications individuelles...

23. Même s'il ne faut pas oublier que les discussions ont parfois été particulièrement âpres pour imposer cette liberté religieuse. Ainsi, la rédaction large de l'article 9 de la Convention européenne des droits de l'homme ne constituait cependant pas une évidence pour tous les États participant à l'élaboration de ce texte: le délégué de la Suède militait pour un traitement dérogatoire autorisant le maintien de législations nationales comportant des règles restrictives pour certaines institutions et fondations religieuses, en justifiant ces régimes dérogatoires par la place traditionnelle occupée par l'église luthérienne dans cet État. De même, la Turquie souhaitait pérenniser certaines mesures applicables à différents ordres musulmans. Ces ajouts ne seront pas acceptés par les autres négociateurs. Voir G. Gonzalez, La Convention européenne des droits de l'homme et la liberté de religion, Paris, Économica, 1997, p. 7 . 
protection dans le cadre plus large de la protection contre l'intolérance au sens large et le racisme. Le champ d'intervention des organes onusiens en matière de protection de la liberté religieuse s'est, en effet, progressivement déplacé: les premiers projets des Nations Unies en matière de protection de la liberté religieuse dans les années 1960 mettaient l'accent, au moins implicitement, sur la nécessité pour les législations nationales de protéger sur une base égalitaire toutes les convictions religieuses ${ }^{24}$. Désormais, les Nations Unies font de la préservation de la diversité religieuse un élément de la lutte contre le racisme au sens large. La Déclaration du Millénaire pose le principe de tolérance comme «l'une des valeurs fondamentales indispensables aux relations internationales au XXI ${ }^{\mathrm{e}}$ siècle ${ }^{25}$. Bien évidemment, la tolérance religieuse et la coexistence entre les différents groupes religieux existant au sein d'un même État s'inscrivent dans cette promotion onusienne de la tolérance. En ce sens, la Commission des droits de l'homme des Nations Unies adopte régulièrement depuis 1999 des textes visant à lutter contre toute diffamation religieuse. La résolution 2001 / 4 vise ainsi à « la lutte contre la diffamation des religions en tant que moyen de promouvoir les droits de l'homme, l'harmonie sociale et la diversité religieuse et culturelle». Avec la résolution 2004/ 6 (« La lutte contre la diffamation des religions»), la Commission des droits de l'homme s'affirme « convaincue que, dans un monde qui s'uniformise, la diversité religieuse et culturelle doit être considérée comme porteuse d'un élément complémentaire de créativité et de dynamisme et non servir de justification à une nouvelle confrontation idéologique et politique». Ces grandes déclarations de principe sont de plus en plus complétées d'incitations visant les États en vue d'obtenir des transformations de leur droit interne. Ainsi, par la résolution 2004/ 36 ( Élimination de toutes les formes d'intolérance religieuse »), la Commission des droits de l'homme « demande instamment aux États de veiller à ce que leurs systèmes constitutionnel et législatif instituent des garanties adéquates et effectives pour assurer à tous, sans distinction, la liberté de pensée, de conscience, de religion et de conviction, notamment des recours effectifs en cas d'atteinte à la liberté de pensée, de conscience, de religion ou de conviction et au droit de pratiquer librement sa propre religion, y compris la liberté de changer de religion ou de conviction ». Si ce type de résolution vise à légitimer les législations et réglementations antiblasphématoires existant dans un grand nombre d'États (dont au premier lieu les États d'Islam ${ }^{26}$ ), il permet également de rappeler le principe du pluralisme religieux. La résolution du 18 avril 2001 amène même la Commission des droits de l'homme à affirmer que «la diffamation des religions constitue l'une des causes de la discorde sociale et [...] entraîne des violations des droits de l'homme et de leurs adhérents ».

\section{B. Des jurisprudences prudentes}

Faute de textes explicites protégeant les religions minoritaires, les jurisprudences supranationales forment un ensemble restreint en la matière. Bien plus encore, ces jurisprudences apparaissent relativement fragiles et parfois non dénuées d'ambiguités. Cette constatation apparaît fondée tant en ce qui concerne l'approche de la Cour et la Commission européenne des droits de l'homme, que du Comité des droits de l'homme.

Depuis l'arrêt Kokkinakis ${ }^{27}$, on sait que la Cour européenne des droits de l'homme fait de la liberté de pensée, de conscience et de religion protégée par l'article 9 de la Convention "l'une des assises d'une "société démocratique" ». Cette liberté figure, dans sa dimension religieuse, parmi les éléments les plus essentiels de l'identité des croyants et de leur conception de la vie, mais elle est aussi un bien précieux pour les athées, les agnostiques, les sceptiques ou les indifférents. Pour la Cour, «il y va du pluralisme - chèrement conquis au cours des siècles - consubstantiel à pareille société». Cette liberté religieuse, telle que protégée par la Cour, relève d'abord du for intérieur, mais elle implique de surcroît, la liberté de «manifester sa religion ». Le témoignage, en paroles et en actes, est lié à l'existence de convictions religieuses. Bien plus encore, le droit à la liberté de religion, tel que l'entend la Convention, exclut toute appréciation de la part de l'État sur la légitimité des croyances religieuses ou sur les modalités d'expression de celles-ci ${ }^{28}$. Ainsi, un système d'autorisation, qui aboutit à imposer des conditions rigides ou même prohibitives à l'exercice de certains cultes, viole l'article 9 de la Convention ${ }^{29}$. L'État peut néanmoins limiter l'exercice de cette liberté, pour un certain nombre de motifs légitimes, prévus par le deuxième paragraphe de cette disposition ${ }^{30}$.

24. Voir, notamment doc. E / 3873/ 1964 et E / CN / 4 / 920.

25. Déclaration du Millénaire, Résolution 55/2 adoptée par l’Assemblée générale des Nations Unies le 8 septembre 2000.

26. La Commission a, par ailleurs, toujours dénoncé les stéréotypes liés à l'Islam et l'association de cette religion avec les violations des droits de l'homme. Voir, notamment, la résolution 2004 / 6 ( «L lutte contre la diffamation des religions »), où la Commission des droits de l’homme est « alarmée par les conséquences négatives que les événements du 11 septembre 2001 continuent d'avoir pour les minorités et les communautés musulmanes dans certains pays non musulmans et par l'image négative que les organes d'information donnent de l'Islam, ainsi que par l'introduction et la mise en application de lois qui établissent expressément une discrimination à l'encontre des musulmans et les prennent pour cibles ».

27. Cour EDH, 19 avril 1993, Kokkinakis c. Grèce, $\$ 12$. On peut d'ailleurs mentionner que cet arrêt portait sur la délimitation de la liberté religieuse d'un croyant appartenant à un groupe minoritaire (en l'espèce les témoins de Jéhovah).

28. Cour EDH, 26 octobre 2000, Hassan et Tchaouch c. Bulgarie, $\$ 78$.

29. Cour EDH, Manoussakis c. Grèce, 29 août 1996. En l'espèce, le système de l'autorisation institué par la loi n ${ }^{\circ} 1363 / 1938$ et le décret des 20 mai/ 2 juin 1939 visaient tous les cultes non orthodoxes et notamment les témoins de Jéhovah.

30. La reconnaissance d'un pluralisme religieux ne signifie évidemment pas l'admission de toutes les pratiques et attitudes liées à ces cultes minoritaires. La Commission européenne des droits de l'homme avait ainsi eu l'occasion de préciser qu'un État peut parfaitement refuser à un détenu bouddhiste l'autorisation de se laisser pousser la barbe et d'obtenir un chapelet pour des motifs visant «la protection de l'ordre public au sens de l'article $9 \$ 2$ ». Décision du 15 février 1965, X. c. Autriche, Annuaire de la Convention européenne des droits de l'homme, t. 8, p. 185. 
Pour la Cour européenne des droits de l'homme, l'État possède donc une véritable obligation générale de respect des différentes convictions et religions et il doit accepter que les individus puissent librement les adopter (et éventuellement modifier leur choix initial). La liberté religieuse apparaît d'autant plus grande que les organes de la Convention se sont toujours gardés de définir ce qu'est une religion, s'en tenant à une approche ouverte. Pour la Commission européenne des droits de l'homme en 1976, « une église est une communauté religieuse organisée, fondée sur une identité ou sur une substantielle similitude de convictions... l'église elle-même bénéficie d'une protection dans sa liberté de manifester sa religion, d'organiser et de célébrer son culte, d'enseigner les pratiques et les rites... ${ }^{31}$. Cette volonté de ne pas être liée par une définition trop contraignante empêche toutefois que ce corpus jurisprudentiel offre une délimitation explicite de la notion de religion minoritaire ${ }^{32}$.

Au-delà même du respect d'une liberté fondamentale, l'État doit également mettre en œuvre les conditions d'exercice d'un véritable exercice plural des religions. Comme la Cour a eu l'occasion de le rappeler fermement dans l'affaire Kokkinakis, l'État possède, en effet, une véritable mission d'organisation et de protection du pluralisme religieux: "dans une société démocratique, où plusieurs religions coexistent au sein d'une même population, il peut se révéler nécessaire d'assortir cette liberté de limitations propres à concilier les intérêts des divers groupes et à assurer le respect des convictions de chacun » (\$34). Ce postulat ne signifie toutefois pas que les États membres de la Convention européenne des droits de l'homme se voient imposer un quelconque modèle préétabli d'organisation entre l'État et les religions ou les groupes de croyants. Pour la Cour européenne, il existe une large marge d'appréciation laissée aux États membres, «notamment pour ce qui est de l'établissement des délicats rapports entre les Églises et l'État » ${ }^{33}$. La Commission européenne des droits de l'homme avait d'ailleurs été amenée en 1981 à déclarer compatible le système de la religion d'État avec les dispositions de l'article 9 de la Convention. Toutefois, un tel mode d'organisation entre l'Église et l'État ne peut conduire à obliger à adhérer à une religion déterminée ${ }^{34}$.

L'absence de sources textuelles explicites aboutit, de manière assez peu surprenante, à ce que la protection des religions minoritaires soit assurée par l'application du principe de non-discrimination. Ainsi pour la Cour euro- péenne des droits de l'homme, une personne ne peut pâtir des conséquences de sa conversion aux témoins de Jéhovah après son mariage dans le règlement de son divorce et l'attribution de la garde des enfants ${ }^{35}$. De même, le Comité des droits de l'homme des Nations Unies rappelle que l'article $26 \mathrm{du}$ Pacte interdit toute discrimination, fondée notamment sur la religion ${ }^{36}$. Dans la communication Waldman c. Canada ${ }^{37}$, le Comité estime ainsi que les différences de traitement établies au Canada au regard du financement public exclusivement prévu pour les écoles catholiques romaines ne repose pas sur des différences de traitement raisonnables et objectives. Si un État partie décide d'instaurer un financement public pour des établissements confessionnels, ceci ne doit pas aboutir à violer le principe de non-discrimination prévu par l'article 26 du Pacte relatif aux droits civils et politiques. D'une manière plus générale, l'existence de cette religion d'État ne doit conduire à aucune discrimination entre pratiquants et non-pratiquants. La Cour européenne des droits de l'homme a également eu l'occasion de rappeler que des conditions de vie discriminatoires appliquées à un groupe de personnes du fait notamment de leur appartenance religieuse (en l'occurrence des Chypriotes grecs dans la partie nord de l'île occupée par la Turquie) peuvent s'analyser en un traitement dégradant ${ }^{38}$.

Mise en exergue d'un pluralisme religieux, protection contre toute discrimination fondée sur son appartenance religieuse : la protection juridictionnelle des religions minoritaires apparaît indéniable. Elle semble même renforcée, car la jurisprudence européenne n’hésite pas à mettre en avant un principe de protection de la «tranquillité religieuse ». La Cour considère, en particulier, que les États disposent du pouvoir de contrôler si un mouvement ou une association poursuit, à des fins prétendument religieuses, des activités nuisibles à la population ou à la sécurité publique ${ }^{39}$. Les autorités se doivent donc d'instaurer un climat propice à la « coexistence pacifique » entre les différentes religions. Ceci ne doit évidemment pas conduire à éliminer la cause des tensions en supprimant le pluralisme, mais bien plutôt à veiller à ce que les groupes concurrents se tolèrent les uns les autres ${ }^{40}$. Ainsi, pour la Commission européenne des droits de l'homme, « dans les pays où la grande majorité de la population adhère à une religion précise, la manifestation des rites et des symboles de cette religion, sans restriction de lieu et de forme, peut constituer une pression sur les étudiants

31. Décision du 8 mars 1976, X. c. Danemark, DR, 5, p. 157.

32. Ceci aboutit toutefois également à ne pas offrir de définition de la religion minoritaire. Ouverts, a priori, à tout type de croyance, les organes de la Convention s'autorisent par contre à refuser le bénéfice de l'application de l'article 9 à des manifestations qui, bien qu'en relation avec une religion ou une croyance, n'en sont pas moins de nature purement commerciale. Voir Commission EDH, décision du 5 mai 1979, requête $\mathrm{n}^{\circ} 7805 / 77$, Église de $^{2}$ scientologie c. Suède, DR, 16, p. 109

33. Cour EDH, 27 juin 2000, Cha'are Shalom Ve Tsedek c. France, $\$ 84$.

34. La Commission avait ainsi reconnue incompatible avec l'article 9 l'obligation faite à une personne de payer un impôt ecclésial dès lors qu'il n'était pas membre de l'église d'État. Commission EDH, décision du 9 mai 1981, requête n ${ }^{\circ} 11581$ / 86, Église de scientologie c. Suède, DR, 56, p. 166.

35. Cour EDH, 23 juin 1993, Hoffmann c. Autriche, $\$ 28 s q$.

36. Constatations du 25 juillet 2001 sur la communication $n^{\circ} 884 / 1999$, Antonina Ignatane c. Lettonie.

37. Communication n ${ }^{\circ}$ 694/1996, Waldman c. Canada, constatations du 3 novembre 1999, Report of the Human Rights Committee, vol. I, doc. A / 54 / 40.

38. Cour EDH, 10 mai 2001, Chypre c. Turquie, $\$ 309$ et 310 .

39. Voir les arrêts Manoussakis et autres c. Grèce, $\$ 40$; Stankov et Organisation macédonienne unie Ilinden c. Bulgarie, $\$ 84$.

40. Cour EDH, 14 décembre 1999, Sérif c. Grèce, $\$ 53$. 
qui ne pratiquent pas ladite religion ou sur ceux adhérant à une autre religion ${ }^{41}$.

Ce souci d'éviter que des revendications ou manifestations excessives émanant de groupes religieux plus ou moins fantaisistes, voire dangereux, ne nuisent au respect de l'ordre public apparaît louable. La volonté proclamée par la Cour d'imposer aux États un principe de neutralité et d'impartialité dans l'exercice de son pouvoir de réglementation dans sa relation avec les divers religions, cultes et croyances ${ }^{42}$ amène toutefois la Cour à développer des distinctions fragiles, voire très contestables : ainsi tentet-elle dans son arrêt Kokkinakis de poser une douteuse distinction entre « le témoignage chrétien » et le «prosélytisme abusif», le premier correspondant à la «vraie évangélisation», le second en représentant « la corruption ou la déformation ». Le juge Pettiti, dans son opinion partiellement concordante, avait d'ailleurs pointé les contradictions d'une telle jurisprudence, en indiquant qu'il est nécessaire d'accepter l'utilisation du prosélytisme, même de mauvaise foi, c'est-à-dire le droit d'exposer ses convictions, de tenter de les faire partager et même de tenter de convertir son interlocuteur ${ }^{43}$. De son côté, le juge Martens, dans une opinion partiellement dissidente, se montrait encore plus clair : «Que quelqu'un envisage ou non de changer de religion ne regarde pas l'État et, en conséquence, que quelqu'un tente d'inciter autrui à changer de religion ne devrait en principe pas le regarder non plus » ${ }^{44}$.

L’argumentaire développé par la Cour européenne des droits de l'homme dans les affaires Otto Preminger Institut ${ }^{45}$ et Wingrove ${ }^{46}$ semble également de nature à laisser planer des doutes quant à la véritable efficacité de la protection des religions minoritaires par la Cour européenne des droits de l'homme ${ }^{47}$. Avec le premier arrêt, la Cour accorde une importance extrême au fait que le catholicisme soit la religion de $87 \%$ du Tyrol et de $78 \%$ de l'Autriche. Ceci aboutit à penser que la répression de propos blasphématoires aurait probablement été appréciée d'une autre manière si ceux-ci avaient été dirigés à l'encontre d'une religion minoritaire. De même, dans l'affaire Wingrove contre Royaume-Uni, la Cour, face à une législation anglaise qui ne réprime que le blasphème attentatoire à la foi chrétienne, estime qu'il ne lui appartient pas « de se prononcer in abstracto sur la compatibilité du droit interne avec la Convention. Le degré de protection assuré par le droit anglais aux autres croyances n'est pas en jeu devant la Cour, laquelle doit se borner à examiner l'affaire dont elle est saisie» (\$ 50). La Cour, tout en soulevant que la loi sur le blasphème ne traitait pas à égalité les différentes religions pratiquées au Royaume-Uni, pouvait parfaitement affirmer dans le même temps qu'une telle discrimination n'enlevait rien à la légitimité du but poursuivi par l'incrimination du blasphème telle que consacrée par la common law. Or, comme l'affirmait le juge Pettiti dans son opinion concordante sous l'arrêt Wingrove, la défense des droits d'autrui telle que protégée par la Convention européenne des droits de l'homme ne peut se réduire à la protection d'une seule catégorie de croyances ou de philosophies, ou d'une majorité de celles-ci.

La combinaison d'une approche large de la liberté de religion, couplée à l'application du critère de non discrimination, aboutit donc à faire de la protection des religions minoritaires un élément indiscutable. Ceci ne saurait toutefois pas occulter que les religions minoritaires bénéficient d'un autre axe de protection, lié à la protection des groupes minoritaires par le droit tant international qu'européen.

\section{La protection des religions minoritaires comme composante de la notion de minorité nationale}

Si la protection des religions minoritaires passe par une conception large et « concurrentielle» de la liberté de religion, on peut également estimer qu'il existe un autre angle de reconnaissance et de protection pour ces groupes et leurs croyants. L'apparition, depuis la Société des Nations, de systèmes de protection des minorités nationales a, en effet, permis de reconnaître l'existence des religions minoritaires comme critère même de définition de certains groupes minoritaires. Cette approche a permis de construire peu à peu un ensemble normatif protecteur des minorités religieuses (A), même si les mécanismes de protection apparaissent encore aujourd'hui particulièrement lacunaires, car cantonnés à des techniques de type diplomatique (B).

\section{A. Un ensemble normatif protecteur des minorités religieuses}

Parmi les grands domaines d'intervention du droit international et européen au $\mathrm{xx}^{\mathrm{e}}$ siècle figure la protection des minorités nationales. La Première Guerre mondiale a considérablement remodelé la carte politique de l'Europe et du Proche-Orient, en entraînant la création de nouveaux États, la renaissance d'États disparus et le démembrement d'autres préexistants (tels que la Pologne,

41. Requête $\mathrm{n}^{\circ} 16278$ / 90, décision du 3 mai 1993, Karaduman c. Turquie. Cette appréciation visait le port du foulard islamique en Turquie.

42. Cour EDH, 26 octobre 2000, Hassan et Tchaouch c. Bulgarie, $\$ 78 ; 5$ décembre 2001, Église métropolitaine de Bessarabie et autres c. Moldova, $\$ 116$.

43. Série A, $\mathrm{n}^{\circ}$ 260-A, p. 26.

44. Ibid., p. $37, \$ 14$

45. Cour EDH, 20 septembre 1994, Otto Preminger Institut c. Autriche, Série A, nº 265-A. Voir P. Wachsmann, «La religion contre la liberté d'expression », Revue trimestrielle des droits de l'homme, 1994, p. 441 sq.

46. Cour EDH, 25 novembre 1996, Wingrove c. Royaume-Uni, \$50. Voir notre commentaire, «La liberté d'expression et le blasphème », Revue trimestrielle des droits de l'homme, 1997, p. 725 sq.

47. Voir, sur ce point, J.-F. Flauss, « La diffamation religieuse », in La Protection internationale de la liberté religieuse, J.-F. Flauss (éd.), Bruxelles, Bruylant, 2002, p. 299. 
la Tchécoslovaquie, la Yougoslavie, la Bulgarie, l'Albanie et la Roumanie). La présence de minorités sur les territoires étatiques a très rapidement été analysée comme une menace par les États concernés. Cette crainte va conduire les pays victorieux (les «Principales Puissances Alliées et Associées ») à persuader les nouveaux États de conclure des traités spéciaux pour protéger leurs minorités et la pression de ces groupes minoritaires eux-mêmes va entrầner la prise de nombreuses normes conventionnelles. Les traités dits de minorités, conclus à partir de 1919, vont leur reconnaître certains droits, mais également les placer sous la garantie de la Société des Nations ${ }^{48}$. Tous ces traités avaient pour perspective la fixation «d'obligations d'intérêt international » transcendant l'intérêt particulier de leurs signataires. Le premier de ces traités a été conclu le 29 juin 1919 à Versailles entre les Principales Puissances Alliées et Associées et la Pologne. Cette convention constitue le traité de référence, qui servira de modèle aux traités conclus postérieurement ${ }^{49}$. Ce texte engage les États à n'exercer aucune discrimination à l'égard des minorités et à leur accorder les droits nécessaires à la préservation de leur identité, ce qui concerne notamment le respect de leur intégrité religieuse. En vertu de l'article 2 de ce traité, le gouvernement polonais s'engage à accorder à tous les habitants pleine et entière protection de leur vie et de leur liberté, sans distinction de naissance, de nationalité, de langage, de race ou de religion. En vertu du deuxième alinéa de cet article 2, "tous les habitants de la Pologne auront droit au libre exercice, tant public que privé, de toute foi, religion, ou croyance, dont la pratique ne sera pas incompatible avec l'ordre public et les bonnes mœurs ». De même, en vertu de l'article 7 , «la différence de religion, de croyance ou de confession ne devra nuire à aucun ressortissant polonais en ce qui concerne la jouissance des droits civils et politiques $[\ldots]$ ». De manière encore plus explicite, l'article 8 prohibe toute différence de traitement fondée notamment sur la religion. Quant aux articles 10 et 11, ils fondent des dispositions protectrices concernant plus spécialement les juifs (responsables de la gestion des fonds publics alloués aux écoles juives, ainsi que de la direction de ces écoles; et protégés contre tout acte quelconque qui serait accompli en violation du Shabbat), sauf en cas « des nécessités du service militaire, de la défense nationale, ou du maintien de l'ordre public» (art.11).

Avec ces différentes conventions apparaît la nécessité de protéger la liberté religieuse des groupes minoritaires, non comme un souci de protection de la tolérance religieuse, mais bien plutôt comme une volonté de protéger l'une des caractéristiques principales des groupes minoritaires. La justice internationale va d'ailleurs retenir une approche comparable. La première définition de la minorité retenue par la Cour permanente de justice internationale, dans son célèbre avis du 31 juillet 1930 sur les communautés gréco-bulgares, fait, en effet, de la religion l'un des critères permettant d'identifier ces groupes humains ${ }^{50}$. Cette même Cour, dans plusieurs avis consultatifs, et notamment celui du 6 avril 1935 sur la question des écoles minoritaires en Albanie ${ }^{51}$, mentionne l'obligation de faire respecter l'identité, notamment religieuse, de ces groupes. Les définitions contemporaines vont également toutes retenir cet élément de religion particulière appliquée au groupe minoritaire. Dans un rapport établi pour les Nations Unies en 1978 (à propos de l'article 27 du Pacte sur les droits civils et politiques), le Professeur Capotorti a ainsi proposé une formulation devenue classique et qui définit comme minorité « un groupe numériquement inférieur au reste de la population d'un État, en position non dominante, dont les membres - ressortissants de l'État - possèdent du point de vue ethnique, religieux ou linguistique des caractéristiques qui diffèrent de celles du reste de la population et manifestent même de façon implicite un sentiment de solidarité, à l'effet de préserver leur culture, leurs traditions, leur religion ou leur langue ${ }^{52}$. En 1984, la sous-commission des droits de l'homme des Nations Unies a donné à J. Deschênes le soin de poser à nouveau la problématique de la définition. Selon lui il s'agit «d'un groupe de citoyens d'un État en minorité numérique et en position non dominante dans cet État, dotés de caractéristiques ethniques, religieuses ou linguistiques différentes de celles de la majorité de la population, solidaires les uns des autres, fût-ce implicitement d'une volonté collective de survie et visant à l'égalité en fait et en droit avec la majorité ${ }^{53}$.

Les différents instruments de protection supranationale des minorités nationales, sans aucune exception, font de la religion un élément central de défense des spécificités minoritaires et ce tant en ce qui concerne les instruments généraux que ceux plus spécifiques.

La disposition la plus connue relative aux minorités au sein d'un texte général de protection est indéniablement encore aujourd'hui l'article 27 du Pacte international relatif aux droits civils et politiques : «Dans les États où il existe des minorités ethniques, religieuses ou linguistiques, les personnes appartenant à ces minorités ne peuvent être

48. Voir J. Verhoeven, «Les principales étapes de la protection des minorités », Revue trimestrielle des droits de l'homme, 1997, p. 177 sq.

49. Tels que les traités sur la protection des minorités au Royaume des Serbes, croates et slovènes (10 septembre 1919), la Tchécoslovaquie (10 septembre 1919), la Roumanie ( 9 décembre 1919), la Grèce (10 août 1920). On peut y ajouter les clauses relatives aux minorités des traités de paix conclus avec l'Autriche (traité de Saint-Germain, 10 septembre 1919), la Hongrie (traité de Trianon, 4 juin 1920) et la Turquie (traité de Lausanne, 24 juillet 1923 ). Voir Protection des minorités de langue, de race et de religion par la Société des Nations, SDN, 1927.

50. La minorité se définissant, en effet, comme une « collectivité de personnes vivant dans un pays ou dans une localité données, ayant une race, une religion, une langue et des traditions qui leur sont propres, et unies par l'identité de cette race, de cette religion et de ces traditions dans un sentiment de solidarité, à l'effet de conserver leurs traditions, de maintenir leur culte, d'assurer l'instruction et l'éducation de leurs enfants conformément au génie de leur race et de s'assister mutuellement ». Avis sur l'interprétation de la convention entre la Grèce et la Bulgarie, relative à l'émigration réciproque (communautés gréco-bulgares), série $\mathrm{B}, \mathrm{n}^{\circ} \mathrm{17}, 1930$.

51. Publications de la CPJI, Série A-B, $\mathrm{n}^{\circ} 64$.

52. F. Caportoti, Study on the rights of persons belonging to ethnic, religious, and linguistic minorities, doc. E / CN.4 / Sub.2 / 384 / Rev. I, 1979.

53. Doc. E / CN.4/Sub.2 / 1985/31, \$181. 
privées du droit d'avoir, en commun avec les autres membres de leur groupe, leur propre vie culturelle, de professer et de pratiquer leur propre religion, ou d'employer leur propre langue ». Aux termes de cette disposition, les personnes appartenant à une minorité se voient donc reconnaître le droit au respect de leur identité, en particulier religieuse, et ont le droit de préserver les caractéristiques qu'elles souhaitent maintenir et développer. Pour le Comité des droits de l'homme, le respect des groupes religieux minoritaires implique l'adoption de mesures positives de la part des États, tels que l'acceptation d'un changement de nom pour des Néerlandais convertis à l'hindouisme ${ }^{54}$.

La même conception est retenue par les instruments internationaux spécialement dédiés à la protection des minorités. Au niveau international, l'année 1992 a vu l'adoption de la Déclaration onusienne sur les droits des personnes appartenant à des minorités nationales ou ethniques, religieuses et linguistiques ${ }^{55}$. Ce texte, adopté rapidement sous la pression des événements survenus en Europe centrale et orientale, est un instrument purement déclaratif, comportant nombre de formulations vagues, et ne contenant pas de mécanisme propre de contrôle. Toutefois, ce très court texte de neuf articles tente un équilibre intéressant entre les droits reconnus aux personnes appartenant à une minorité et permettant de développer leur identité et leurs caractéristiques, et les obligations correspondantes des États aux fins de préserver l'intégrité territoriale et l'indépendance politique. Aux termes de cette Déclaration, les États doivent protéger l'existence et l'identité des personnes appartenant à des minorités, en particulier en matière religieuse $\left(\operatorname{art} .1^{\text {er }}\right)$. Les personnes appartenant à ces minorités ont donc le droit de pratiquer leur propre religion (art. $2 \$ 1$ ) et le droit de participer à la vie religieuse ( $\operatorname{art.} 2 \$ 2$ ).

La convention cadre du Conseil de l'Europe pour la protection des minorités nationales adoptée par le Comité des ministres du Conseil de l'Europe le 10 novembre 1994 fait également de la religion l'un des droits minoritaires protégés. Ce texte constitue le seul instrument multilatéral juridiquement contraignant consacré uniquement à la protection des minorités nationales en général. Ce texte européen reprend la technique de la « convention cadre » qui contient pour l'essentiel des dispositions-programmes, définissant certains objectifs que les parties s'engageront à poursuivre. Ces dispositions, qui ne sont pas directement applicables, laissent aux États concernés une marge d'appréciation dans la mise en œuvre des objectifs qu'ils se sont engagés à atteindre et permettront ainsi à chacun d'entre eux de tenir compte de situations parti- culières ${ }^{56}$. Les parties s'engagent ainsi «à promouvoir les conditions propres à permettre aux personnes appartenant à des minorités nationales de [...] préserver les éléments essentiels de leur identité que sont leur religion, leur langue, leurs traditions et leur patrimoine culturel» (art. 5) et à « reconnaître à toute personne appartenant à une minorité nationale le droit de manifester sa religion ou sa conviction, ainsi que le droit de créer des institutions religieuses, organisations et associations » (art. 8). Cette dernière disposition ne définit ni le contenu ni les modalités d'exercice de cette liberté. Cette situation n'est nullement fortuite, car elle exprime la volonté des auteurs de se référer à l'article 9 de la Convention européenne des droits de l'homme et à l'interprétation qui en est donnée par la jurisprudence de la Cour. La liberté religieuse des minorités est ainsi entendue de manière très large, ce qui permet de couvrir tout à la fois l'exercice du culte, les pratiques et rites religieux, ainsi que l'enseignement de la religion.

Les normes, tant internationales qu'européennes, visent à assurer une protection effective de la religion en tant qu'élément essentiel de la notion de minorité nationale. Toutefois, cette pluralité des sources normatives ne saurait masquer le déficit de structures et de mécanismes de protection qui restent, encore aujourd'hui, assez largement déficients car limités à des techniques de type diplomatique.

\section{B. Des techniques de protection de nature diplomatique}

Les textes supranationaux de protection des minorités nationales présentent un point commun : ces normes, qu'elles soient européennes ou internationales, ne sont assorties d'aucun mécanisme contraignant de surveillance, ce qui exclut évidemment toute perspective d'une protection assurée par des techniques juridictionnelles.

Les institutions onusiennes ont choisi, depuis les années 1980, de retenir une approche sectorielle de protection des droits de l'homme, en multipliant les procédures dites ad hoc. La protection des minorités utilise aujourd'hui cette technique de protection au plan international. Le caractère ad hoc de ces procédures spéciales est sensé permettre de répondre de façon plus souple aux graves violations des droits de l'homme que les organismes de traité (la protection des minorités nationales ne faisant de toute manière pas l'objet d'un texte de nature conventionnelle au plan international). Les experts chargés de

54. Les requérants indiquaient que pour toute personne souhaitant étudier et pratiquer les préceptes de la religion hindoue et devenir prêtres, il est obligatoire d'adopter des noms hindous. Pour le Comité, le refus opposé aux requérants constitue une violation de l'article $17 \$ 1$ du Pacte sur les droits civils et politiques. Dans son opinion dissidente, M. Nisuke Ando estime pour sa part qu'un tel changement de nom justifié par l'adhésion à une religion minoritaire rentre très probablement dans le cadre des limitations à l'exercice de la liberté de religion telle que prévue par l'article $18 \$ 3$. Communication $\mathrm{n}^{\circ}$ 453/1991, A.R. Coeriel and M.A.R. Aurik c. Pays-Bas, 31 octobre 1994; International Covenant on civil and political rights, Selected decisions of the Human Rights Committee, vol. 5, forty-seventh to fifty-fifth sessions, United sessions, New York - Genève, 2005, p. 72.

55. Résolution 47 / 135 adoptée par l'Assemblée générale des Nations Unies le 18 décembre 1992.

56. La faiblesse évidente du libellé de la Convention tient en effet pour une grande part aux multiples conditions et réserves incluses dans ses dispositions, telles que : «lorsque ces personnes en font la demande et que celle-ci répond à un besoin réel» (art. 10 $\$ 2$ ), «lorsqu’il y a une demande suffisante» (art. $11 \S 3$ ), «s'il existe une demande suffisante» ( art. $14 \$ 2$ ), etc. Toutefois, l'article 2 prévoit que ces conditions doivent être interprétées « de bonne foi ", c'est-à-dire qu'elles ne doivent pas servir à refuser les exigences des minorités mais au contraire à les prendre en compte. 
ces mandats spéciaux relatifs aux droits de l'homme agissent à titre individuel et sont appelés rapporteur ou représentant spécial, ou expert indépendant. Ils examinent, surveillent et rendent compte publiquement à l'autorité onusienne dont ils dépendent, soit de la situation des droits de l'homme dans des pays ou territoires spécifiques (mandats « par pays »), soit de problèmes d'ensemble qui entraînent de graves violations des droits de l'homme partout dans le monde (mandats dits «thématiques»). Par sa résolution 1986 / 20 du 10 mars 1986, la Commission des droits de l'homme a décidé de nommer un rapporteur spécial sur l'intolérance religieuse ${ }^{57}$ chargé d'examiner «les incidents et mesures gouvernementales, dans le monde entier, incompatibles avec les dispositions de la Déclaration de 1981 sur l'élimination de toutes les formes d'intolérance et de discrimination fondées sur la religion ou la conviction » et « de recommander les mesures à prendre pour remédier aux situations ainsi créées, notamment, selon qu'il conviendra, l'encouragement d'un dialogue entre les communautés confessionnelles ou les groupes de croyants et les gouvernements de leur pays ». Le mandat a été renouvelé sans interruption depuis sa création ${ }^{58}$. La mission de ce rapporteur spécial relève donc d'une mission d'enquête approfondie, puisque son mandat apparaît particulièrement large. Il est notamment chargé d'observer « la façon dont les États s'acquittent de leurs obligations » en matière de tolérance religieuse (phrase pouvant apparaître choquante étant entendu que les États, en la matière, ne sont liés par aucun dispositif conventionnel). Cette surveillance s'opère à l'égard des normes étatiques, des garanties et des recours, mais vise également certains éléments plus précis tels que la protection de la sûreté des personnes et la prohibition de la torture et des arrestations arbitraires pour des motifs religieux. Le contrôle porte enfin sur le respect des lieux de culte et lieux saints, et, d'une manière générale, cherche à s'assurer qu'il n'existe pas dans les États de discriminations fondées sur des motifs liés à l'exercice d'une religion particulière.

Ce rapporteur spécial utilise, pour mener à bien sa mission générale de surveillance, une combinaison de plusieurs techniques de contrôle et d'investigation.

La technique la plus efficace semble être celle de la vérification sur place des atteintes aux droits des minorités religieuses. Or, ce type de contrôle in situ s'avère visi- blement fort délicat à mener. M. Amor n'a ainsi pu effectuer que seize visites en onze ans de mandat, sept États ayant implicitement ou explicitement refusé le principe d'une telle visite sur leur territoire ${ }^{59}$. Pour 2005, l'Azerbaïdjan, le Bangladesh, l'Érythrée, l'Iran, le Kirghizistan, le Nigeria, l'Ouzbékistan, le Sri Lanka étaient pressentis pour faire l'objet d'une visite. Or, seuls le Nigeria, le Bangladesh et le Sri Lanka avaient répondu favorablement fin 2004 et s'étaient déclarés disposés à accueillir $\mathrm{M}^{\mathrm{me}}$ Asma Jahangir dans le courant de l'année $2005^{60}$. L'exercice des visites sur place constitue un exercice sensible, ce qui explique les précautions diplomatiques parfois prises avant leur mise en place. Ainsi en 2004, le rapporteur spécial «rappelle que ces demandes de visite ne représentent pas une appréciation préétablie ou l'expression d'un jugement négatif à l'encontre des gouvernements concernés. Il s'agit, par le biais de visites, d'établir ou d'approfondir le dialogue avec les autorités et l'ensemble des parties concernées, en particulier les organisations non gouvernementales et tous les individus ayant un intérêt particulier pour le mandat ${ }^{61}$.

En pratique l'essentiel de l'action menée par le rapporteur spécial passe par des «communications» adressées aux États, chaque fois que sont portés à sa connaissance des situations ou des cas particulièrement critiques dans un pays donné. Cette technique a connu un essor considérable depuis 2000, car elle est également devenue un moyen de suivi régulier, et non plus seulement ponctuel, de problèmes sérieux dans un État particulier. Depuis 1994, les rapporteurs spéciaux pratiquent également les " appels urgents », dont le nombre reste limité, afin de répondre de manière plus efficace et plus rapide à des situations et des cas très graves ${ }^{62}$. Les différents rapports produits par les rapporteurs spéciaux de l'Organisation des Nations Unies présentent un constat souvent précis et détaillé des violations de la liberté religieuse des minorités, des atteintes aux personnes, aux lieux de cultes, ou aux publications religieuses. Plus qu'une institution permettant de résoudre les problèmes liés aux atteintes portées aux droits religieux, le rapporteur spécial permet de dresser un tableau exhaustif des atteintes portées aux droits des religions minoritaires dans la communauté internationale. L'analyse des différentes communications a ainsi permis d'établir sept catégories d'atteintes au pluralisme

57. La Commission des droits de l'homme a modifié en 2000 (résolution 2000 / 33) le titre du rapporteur spécial sur l’intolérance religieuse, qui est devenu le rapporteur spécial sur la liberté de religion ou de conviction.

58. Dans sa résolution 2004/36 du 19 avril 2004, la Commission des droits de l'homme a encouragé le rapporteur spécial à poursuivre ses efforts et a prorogé de trois ans son mandat. Depuis 1986 se sont succédés à ce poste MM. Angelo d'Almeida Ribeiro (1986-1993), M. Abdelfattah Amor (1993-2004) et $\mathrm{M}^{\mathrm{me}}$ Asma Jahangir (depuis 2004).

59. Les États ayant refusé le principe d’une visite durant cette période sont l’Indonésie, Maurice, Israël, la Fédération de Russie, la République populaire démocratique de Corée, le Nigeria et le Turkménistan.

60. Rapport soumis par la rapporteuse spéciale sur la liberté de religion ou de conviction, Asma Jahangir, doc. E / CN.4/ $2005 / 61,20$ décembre 2004.

61. Rapport soumis par le rapporteur spécial sur la liberté de religion ou de conviction, Abdelfattah Amor, doc. E/ CN.4/ $2004 / 6,16$ janvier 2004 , \$ 6.

62. Sur l'année 2004, 69 communications ont été adressées à 38 États. 4 de ces communications ont revêtu le caractère d' «appel urgent ». Les gouvernements de la Chine, de l'Indonésie, de l'Irak, d’Israël, du Kazakhstan, de la Mongolie, du Myanmar, du Nigeria, de la République de Moldova, de la République unie de Tanzanie, de la Serbie et Monténégro et du Soudan n’ont répondu à aucune des communications adressées. Trois appels urgents et deux lettres faisant état d'allégations ont été envoyés conjointement avec d'autres procédures spéciales comme le rapporteur spécial sur les exécutions extrajudiciaires, sommaires ou arbitraires, le rapporteur spécial sur la promotion et la protection du droit à la liberté d'opinion et d'expression, le rapporteur spécial sur le droit qu'a toute personne de jouir du meilleur état de santé physique et mental possible, le rapporteur spécial sur la violence contre les femmes, ses causes et ses conséquences, le rapporteur spécial sur l'indépendance des juges et des avocats et le rapporteur spécial sur la question de la torture. Doc. E / CN.4 / 2005 / 61, p. 12-13. 
religieux : atteintes au principe de non-discrimination dans le domaine de la religion à l'encontre de certaines communautés; atteintes au principe de tolérance dans le domaine de la religion ou de la conviction; atteintes à la liberté de pensée, de conscience, de religion ou de conviction; atteintes à la liberté de manifester sa religion ou sa conviction; atteintes à la liberté de disposer de biens religieux; atteintes au droit à la vie, à l'intégrité physique et à la santé des personnes (religieux, croyants et noncroyants); atteintes affectant les femmes ${ }^{63}$.

Les conclusions du rapport 2004 montrent cependant les limites de l'action de cette institution : « la rapporteuse spéciale a constaté que l'absence de liberté de religion ou de conviction ou sa limitation demeure une réalité pour un grand nombre d'individus de par le monde. À cet égard, elle entend tout faire pour que, dans l'exercice de son mandat, elle soit informée de la plupart de ces violations et puisse prendre les mesures appropriées $"{ }^{64}$. Cette bonne volonté affichée laisse toutefois planer de sérieux doutes sur l'existence de telles « mesures appropriées »...

Au niveau européen, la mise en œuvre de la convention cadre pour la protection des minorités nationales relève de modalités de contrôle certes plus formalisées, mais qui renvoient également à une approche largement diplomatique. C'est au Comité des ministres du Conseil de l'Europe qu'incombe la tâche générale de veiller à la mise en ouvre de la convention cadre par les parties contractantes. On peut toutefois douter du rôle de cette institution, organe politique composé des ministres des Affaires étrangères des États membres, qui reçoivent souvent les instructions de leur capitale et s'efforcent de parvenir à des consensus autour des décisions adoptées. Mais l'efficacité du suivi de la mise en ouvre de la convention cadre relève surtout d'un comité consultatif de dix-huit membres, composé d'experts indépendants. Sa tâche est d'assister le Comité des ministres lorsque celui-ci évalue l'adéquation des mesures prises par une partie pour donner effet aux principes énoncés par la convention. Dans ces avis, le Comité n'agit pas comme un juge, mais cherche davantage à faciliter une meilleure application du texte. Il tient compte du fait que la convention cadre est un instrument souple, mais qui n'en fixe pas moins des normes juridiques concernant la protection des minorités nationales. En outre, le dialogue avec les États est assuré par ce que l'on appelle les «séminaires de follow-up», qui réunissent les autorités, la société civile ainsi que des représentants du Comité consultatif afin d'examiner, sur place, comment mettre à exécution les recommandations des instances de suivi de la convention cadre.
Comme au plan international, les rapports étatiques permettent d'obtenir un panorama assez complet de la situation des religions minoritaires dans les trente-sept États parties à la convention cadre. Les rapports périodiques doivent, en effet, décrire la situation de l'État partie en ce qui concerne l'application de l'article 8 de la convention (en présentant tout à la fois le cadre juridique, les infrastructures d'État et les mesures prises). Le rapport doit également mentionner s'il existe une religion d'État dans le pays (et si cela est le cas si cette religion est définie juridiquement) et préciser si la loi énumère les religions reconnues. Mais les avis adoptés à l'encontre des États (lorsque ceux-ci sont rendus publics) restent souvent marqués par une extrême prudence. Ainsi lorsque le comité consultatif se préoccupe de la conformité du système de soutien apporté par l'État à l'Église nationale danoise avec l'égalité de traitement par rapport aux autres religions, il se contente de demander aux autorités danoises d' « examiner cette question »... ${ }^{65}$. De même face à des discriminations frappant les musulmans en Moldova, le Comité ne peut qu'indiquer que cet État « devrait prendre toutes les mesures nécessaires, sur le plan juridique, administratif ou autre, afin de s'assurer que les personnes appartenant aux minorités nationales de confession musulmane bénéficient sans entrave injustifiée et sans discrimination du droit de manifester leur religion et de créer des organisations religieuses, en conformité avec l'article 8 de la convention cadre ${ }^{66}$. La prudence du Comité l'amène même parfois à utiliser des formulations dont la clarté n'est pas la qualité première... Ainsi, face à l'existence de plusieurs minorités ayant une religion différente de la grande majorité de la population polonaise, le Comité consultatif «rappelle la nécessité, pour les autorités, de prendre suffisamment en compte cette dimension dans l'élaboration de mesures et de politiques en faveur de ces minorités nationales, y compris en ce qui concerne la distribution d'aides financières $» . .{ }^{67}$. On peut même s'étonner que le Comité, examinant la situation d'États où les tensions entre religions sont extrêmes, se contente d'un examen tout à fait sommaire et insuffisant de l'application de l'article 8 dans cet État partie ${ }^{68}$. Il est, d'une manière générale, extrêmement délicat d'analyser la portée exacte du travail du Comité des ministres et du Comité d'experts sur la protection des minorités religieuses en Europe (en particulier parce que la protection de ces groupes doit être prise en compte dans le cadre plus large du contrôle opéré sur les États membres par toutes les institutions du Conseil de l'Europe). S'il est indéniable que ces institutions effectuent un travail de contrôle et de surveillance qui

63. Voir, notamment, le rapport soumis par le rapporteur spécial sur la liberté de religion ou de conviction, Abdelfattah Amor, 16 janvier 2004. Doc. E/ CN.4/2004/63, \$115 sq.

64. Doc. E/CN.4/ 2005/61, précité, \$74.

65. Deuxième avis sur le Danemark, adopté le 9 décembre 2004. Doc. ACFC / INF / OP / II (2004) 005, \$25.

66. Deuxième avis sur la Moldova, adopté le 9 décembre 2004. Doc. ACFC / INF / OP / II (2004) $004 \$ 81$. Le même type de constatation a été effectué concernant la situation des musulmans en Croatie. Deuxième avis sur la Croatie, adopté le $1^{\text {er }}$ octobre 2004. Doc. ACFC/INF / OP / II (2004) o02, \$37 sq.

67. Avis sur la Pologne du 27 novembre 2003. Doc. ACFC / INF / OP / I (2004) 005, \$59.

68. Tel est notamment le cas pour la Bosnie-Herzégovine, où le Comité se contente en 2004, en deux brefs paragraphes, de "prendre acte » d'une nouvelle loi sur la liberté de religions et le statut juridique des Églises et d'exprimer sa «préoccupation » devant les divers obstacles s'opposant à la reconstruction des édifices religieux. Avis sur la Bosnie-Herzégovine du 27 mai 2004. Doc. ACFC / INF / OP / I (2005) 003, \$74 et 75. 
semble effectif, on peut néanmoins douter des répercussions de ces avis et recommandations sur les États membres. À cet égard, on peut noter que la Cour européenne des droits de l'homme s'avère particulièrement prudente dans son analyse de la convention cadre. Face à une requérante lui demandant de prendre en compte « instamment » ce texte, illustration d'un consensus international qui permettrait de mettre en lumière la nécessité de protection de groupes particuliers (tels que les tziganes), la Cour indique qu'elle «n'est pas convaincue que ce consensus soit suffisamment concret pour qu'elle puisse en tirer des indications quant au comportement ou aux normes que les États contractants considèrent comme souhaitables dans une situation donnée. La convention cadre, par exemple, énonce des principes et objectifs généraux, mais les États signataires n'ont pas été en mesure de s'accorder sur les modalités de sa mise en œuvre ${ }^{69}$.

La protection des religions minoritaires par le droit international et européen apparaît donc comme un ensemble imparfait, qui peine à dépasser le stade normatif. Si l'on constate tout à la fois un déclin progressif des politiques antireligieuses et du contrôle total du religieux au nom d'une idéologie politique de la part des États, si l'on observe l'essor fragile de tentatives de rapprochement entre religions, ceci ne saurait occulter la multiplication des exemples d'intolérance religieuse. Le développement de l'extrémisme (quelle que soit la religion considérée) aboutit régulièrement à des actes de violence perpétrés sur ceux qui n'appartiennent pas au même camp. Les différents systèmes de protection des religions minoritaires s'accordent d'ailleurs à relever une croissance continue de l'islamophobie. Ceci démontre que dans de très nombreux cas les États manquent à leurs obligations positives de protection des personnes de toutes confessions ou convictions se trouvant sous leur juridiction contre les atteintes portées à leur droits, y compris lorsqu'elles sont commises par des acteurs ou des entités non étatiques. Les institutions nationales se montrent souvent peu empressées à protéger les sanctuaires religieux, ce qui ne permet pas d'entraver l'augmentation des actes de profanation et autres formes de destruction. À cet égard, le maintien des stéréotypes et des clichés apparaît malheureusement hors de portée du droit international et européen. Si le xxI ${ }^{\mathrm{e}}$ siècle "sera religieux ou ne sera pas ", selon la phrase attribuée à $\mathrm{A}$. Malraux, il est à craindre que ce nouveau siècle peine à inscrire durablement la protection des religions minoritaires dans une conception ouverte et tolérante de la liberté religieuse. 